\title{
Extraction of Stewart Atoms from Electron Densities
}

\author{
Peter M. W. Gill ${ }^{\dagger}$ \\ Department of Chemistry, Massey University, Palmerston North, New Zealand
}

Received: May 24, $1996^{\otimes}$

\begin{abstract}
Stewart atoms are the unique nuclear-centered spherical functions whose sum best fits a molecular electron density in a least-squares sense. It is difficult, however, to express Stewart atoms in closed form. We therefore introduce new closed-form approximations, Stewart-Slater atoms, and show that these can be found efficiently from molecular multipole moments. Using examples, we argue that the parameters of Stewart-Slater atoms can yield insight into the nature of chemical bonding.
\end{abstract}

\section{Introduction}

Because the Schrödinger equation for a molecule makes no reference to the constituent atoms, it is generally acknowledged ${ }^{1}$ that it is not possible to derive the atom-in-a-molecule concept from the basic postulates of quantum mechanics. Notwithstanding this, chemists have (and have always had) an overpowering need to rationalize their chemistry in atomic terms. To quote Parr and Yang, "Chemistry is the science of why particular atoms behave in particular ways, and why also do particular functional groups. Combination does not destroy atomic identity; it only perturbs it slightly, or a little more. An atom here is not the same as an atom there, but it is almost so." The same authors also point out that "For molecules, at first sight, densities look like superposed atomic densities; on closer inspection, modest (but still quite small in absolute terms) buildups of density are seen in bonding regions."

Accordingly, over the past few decades, chemists have examined numerous schemes ${ }^{2-24}$ that partition a molecule into its "atomic components". Every scheme begins with the critical recognition that a partition is possible only after the basic postulates have been augmented by an additional assumption and, although each invokes a different assumption, one must concede in the final analysis that any partition is arbitrary and that its merit can be assessed only in terms of its aesthetic purity, its computational cost, and the usefulness of the resulting model.

According to density functional theory, ${ }^{2}$ all quantities of interest may be deduced from the molecular charge density $\rho(\mathbf{r})$. Of course, the density in a real molecule is a complicated function and it would be helpful to be able to decompose it into simple, atom-centered pieces. This is another significant motivation for seeking a meaningful partition of the density.

Of the various competing definitions of atoms in molecules, Bader's partition ${ }^{15}$ based on the zero-flux surfaces of $\rho(\mathbf{r})$ is undoubtedly one of the most popular. Bader has shown that his atoms possess a wide range of useful properties ${ }^{21}$ and his group have made available a practical computer program, ${ }^{11}$ PROAIM, that implements their partitioning scheme. The cost of computing Bader atoms, however, is relatively high ${ }^{16}$ and the Bader approach has hitherto been restricted to moderatesize molecules.

Analogous comments can be made about the charge of an atom in a molecule. For reasons which are closely related to those of the foregoing paragraph, this too defies derivation from quantum mechanical first principles. Indeed, the difficulties here

$\dagger$ Present address: Department of Chemistry, University of Cambridge, Cambridge, CB2 1EW, England.

${ }^{\otimes}$ Abstract published in Advance ACS Abstracts, September 1, 1996. are probably even greater because, even if it were assumed that one could discern the "atoms" in a molecule, it is still not necessarily clear how one would proceed thence to determine the "atomic charges". Although it could be argued that the atomic charge should be the integral over all space of the appropriate atom, this is meaningful only if the atom is compactly localized about its nucleus. In an excellent review, ${ }^{13}$ Hall and Smith survey the progress that has been made but conclude that "the goal of defining an atomic charge, within a molecule, which can be calculated readily and used in discussions of molecular bonding and reactivities, has not yet been reached".

In the present paper, we develop a partitioning scheme based on a remarkable result due to Stewart. , $^{5,8,23}$ The scheme is conceptually simple and yields chemically useful "atoms" at a modest computational cost. Furthermore, it has interesting connections to the novel Gaussian molecular shape model that has been described recently in the work of Grant and Pickup. ${ }^{24}$ We have implemented the scheme within the Q-CHEM program $^{25}$ and all results reported in this paper are in atomic units.

\section{Exact Stewart Atoms}

Consider an $N$-atom molecule whose $j$ th nucleus is at $\mathbf{A}_{j}$ and whose charge density $\rho(\mathbf{r})$ is known. The internuclear distances are $R_{i j}=\left|\mathbf{A}_{i}-\mathbf{A}_{j}\right|$. Stewart's assumption is that the $j$ th "atom" in the molecule is a spherically symmetric function $\sigma_{j}$ centered on the $j$ th nucleus. The functions $\sigma_{j}$, for which we propose the name "Stewart atoms", are defined as those whose sum best fits $\rho(\mathbf{r})$ in a least-squares sense.

The Stewart atoms $\sigma_{j}$ for a given $\rho(\mathbf{r})$ minimize the residual functional

$$
\begin{aligned}
Z(\vartheta)=\iint\left[\rho\left(\mathbf{r}_{1}\right)-\sum \sigma_{j}\left(r_{1 j}\right)\right] \vartheta\left(r_{12}\right) \times \\
\\
{\left[\rho\left(\mathbf{r}_{2}\right)-\sum \sigma_{j}\left(r_{2 j}\right)\right] \mathrm{d} \mathbf{r}_{1} \mathrm{~d} \mathbf{r}_{2} }
\end{aligned}
$$

where $r_{12}=\left|\mathbf{r}_{1}-\mathbf{r}_{2}\right|, r_{1 j}=\left|\mathbf{r}_{1}-\mathbf{A}_{j}\right|$ and the two-electron operator $\vartheta\left(r_{12}\right)$ determines the fit model. The most important models in practice are $\vartheta(\mathrm{r}) \equiv \delta(r), r^{-1}$ or $-r^{+1}$ and these can be shown to fit $\rho(\mathbf{r})$ itself, the electric field due to $\rho(\mathbf{r})$, or the electric potential due to $\rho(\mathbf{r})$, respectively. ${ }^{26}$ We will call the resulting $\sigma_{j}$ density-derived, field-derived, and potential-derived Stewart atoms. Stewart then uses the convolution theorem to rewrite (2.1) in terms of Fourier transforms as

$$
Z(\vartheta)=\int F_{\vartheta}(x)\left|F_{\rho}(\mathbf{x})-\sum F_{j}(x) \mathrm{e}^{i \mathbf{x} \cdot \mathbf{A}_{j}}\right|^{2} \mathrm{~d} \mathbf{x}
$$

Using the calculus of variations to find the $F_{j}(x)$ that minimize 
$Z(\vartheta)$ yields the linear system

$$
\mathbf{J F}=\mathbf{P}
$$

where, after integrating over $\Omega$ (the angular part of $\mathbf{x}$ ), we have

$$
\begin{gathered}
J_{i j}(x)=j_{0}\left(R_{i j} x\right)=\sin \left(R_{i j} x\right) / R_{i j} x \\
P_{j}(x)=\int F_{\rho}(\mathbf{x}) \mathrm{e}^{-i \mathbf{x} \cdot \mathbf{A}_{j}} \mathrm{~d} \Omega=\int \rho(\mathbf{r}) j_{0}\left(r_{j} x\right) \mathrm{d} \mathbf{r}
\end{gathered}
$$

After solving (2.3) for the $F_{j}(x)$, the Stewart atoms can be obtained by the back-transformation

$$
\sigma_{j}(r)=\frac{1}{2 \pi^{2}} \int_{0}^{\infty} x^{2} j_{0}(r x) F_{j}(x) \mathrm{d} x
$$

Details of the extraction of Stewart atoms from a diatomic molecule are given below.

Curiously, the fitting operator $\vartheta$ disappears during the variation process and, as a result, the $\sigma_{j}$ are independent of it. The density-derived, field-derived, and potential-derived Stewart atoms from a given density are therefore all identical, a fact that suggests to us that the Stewart decomposition of the molecular electronic density is a very natural one indeed.

We note that Stewart atoms, unlike Bader atoms, have no boundaries. This is important conceptually for it implies that Stewart atoms overlap and may be regarded as radially perturbed free atoms. Alternatively, Stewart atoms could be interpreted as being "soft" modifications of the traditional "hard-sphere" space-filling models of molecular structure. In either picture, they represent an extension of the "soft Gaussian atoms" recently introduced by Grant and Pickup. ${ }^{24}$

If desired, the "Stewart charge" $\sigma_{j}^{(0)}$ can be found by integrating the Stewart atom, i.e.

$$
\sigma_{j}^{(0)}=\int \sigma_{j}(r) \mathrm{d} \mathbf{r}=F_{j}(0)
$$

and Stewart himself has derived formulas ${ }^{23}$ for these in small systems of various symmetries. However, it is important to realize that, whereas the $\sigma_{j}$ are optimal in a least-squares sense, the $\sigma_{j}^{(0)}$ are not. In particular, if a $\sigma_{j}$ is poorly localized about its nucleus, the associated $\sigma_{j}^{(0)}$ is of little physical significance. However, the Stewart charges possess some attractive features. It can be shown, ${ }^{23}$ for example, that the Stewart charges (placed at their parent nuclei) exactly reproduce the multipole moments of $\rho(\mathbf{r})$ up to a certain multipole order. This property is very desirable. Indeed, in a recent review, ${ }^{22}$ Ángyán and Chipot cited it as the most important to be satisfied by a charge distribution model.

To illustrate the extraction of exact Stewart atoms, we consider the case of a diatomic molecule with nucleus 1 at the origin and nucleus 2 at $\mathbf{R}$. For simplicity, we will assume that $\rho(\mathbf{r})$ is a sum of normalized spherical Gaussians whose centers lie along the bond axis, i.e.

$$
\begin{gathered}
\rho(\mathbf{r})=\sum_{k} U_{k}\left(\frac{\zeta_{k}}{\pi}\right)^{3 / 2} \exp \left(-\zeta_{k}\left|\mathbf{r}-\alpha_{k} \mathbf{R}\right|^{2}\right) \\
F_{\rho}(\mathbf{x})=\sum_{k} U_{k} \exp \left(i \alpha_{k} \mathbf{x} \cdot \mathbf{R}-x^{2} /\left(4 \zeta_{k}\right)\right)
\end{gathered}
$$

It follows from (2.3)-(2.5) that the Fourier transforms of the Stewart atoms are given by

$$
\begin{aligned}
& {\left[\begin{array}{l}
F_{1}(x) \\
F_{2}(x)
\end{array}\right]=\left[\begin{array}{ll}
1 & j_{0}(R x) \\
j_{0}(R x) & 1
\end{array}\right]^{-1} \times } \\
& {\left[\begin{array}{l}
\sum U_{k} j_{0}\left(\alpha_{k} R x\right) \exp \left(-x^{2} /\left(4 \zeta_{k}\right)\right) \\
\sum U_{k} j_{0}\left(\bar{\alpha}_{k} R x\right) \exp \left(-x^{2} /\left(4 \zeta_{k}\right)\right)
\end{array}\right] }
\end{aligned}
$$

where $\bar{\alpha}_{k}=1-\alpha_{k}$. Substituting $F_{1}(x)$ into (2.6) then yields the exact Stewart atom

$$
\begin{gathered}
\sigma_{1}(r)=\sum_{k} \frac{U_{k}}{2 \pi^{2}} \int_{0}^{\infty} \frac{\left[j_{0}\left(\alpha_{k} R x\right)-j_{0}(R x) j_{0}\left(\bar{\alpha}_{k} R x\right)\right]}{1-j_{0}^{2}(R x)} \times \\
x^{2} j_{0}(r x) \exp \left(\frac{-x^{2}}{4 \zeta_{k}}\right) \mathrm{d} x
\end{gathered}
$$

By solving (2.10) in the limit of small $x$, it can be shown that the Stewart charges are

$$
\left[\begin{array}{c}
\sigma_{1}^{(0)} \\
\sigma_{2}^{(0)}
\end{array}\right]=\left[\begin{array}{l}
F_{1}(0) \\
F_{2}(0)
\end{array}\right]=\left[\begin{array}{l}
\sum U_{k} \bar{\alpha}_{k} \\
\sum U_{k} \alpha_{k}
\end{array}\right]
$$

Although the Stewart charges successfully reproduce the charge and dipole moment of $\rho(\mathbf{r})$, the fact that they are completely independent of the Gaussian exponents $\zeta_{k}$ is unsatisfactory and supports the remark above that the Stewart charges are physically significant only if the Stewart atoms are well localized about their respective nuclei.

\section{Stewart Moments and Stewart-Slater Atoms}

Unfortunately, although the foregoing derivation seems to yield exact Stewart atoms, the back-transformation (2.6) yields integrals that are difficult or impossible to handle analytically. We have been unable to solve the integral (2.11), for example, without resorting to quadrature and even our numerical techniques are expensive and unreliable at large $r$. A less ambitious approach is to seek the low-order radial moments of the $\sigma_{j}$. (We note that Stewart himself has used the second moments to estimate atomic sizes in several molecules. ${ }^{23}$ ) We propose that the $\sigma_{j}$ can then be approximately reconstructed from these as described below.

The power series expansions of the elements of $\mathbf{J}, \mathbf{F}$, and $\mathbf{P}$

$$
\begin{gathered}
J_{i j}(x)=1-\frac{R_{i j}{ }^{2}}{3 !} x^{2}+\frac{R_{i j}^{4}}{5 !} x^{4}-\ldots \\
F_{j}(x)=\sigma_{j}^{(0)}-\frac{\sigma_{j}^{(2)}}{3 !} x^{2}+\frac{\sigma_{j}^{(4)}}{5 !} x^{4}-\ldots \\
P_{j}(x)=\rho_{j}^{(0)}-\frac{\rho_{j}^{(2)}}{3 !} x^{2}+\frac{\rho_{j}^{(4)}}{5 !} x^{4}-\ldots
\end{gathered}
$$

involve the internuclear distances $R_{i j}$, the radial moments of the $\sigma_{j}$

$$
\sigma_{j}^{(n)}=\int r^{n} \sigma_{j}(r) \mathrm{d} \mathbf{r}
$$

and the radial moments of $\rho$ about the various nuclei

$$
\rho_{j}^{(n)}=\int\left|\mathbf{r}-\mathbf{A}_{j}\right|^{n} \rho(\mathbf{r}) \mathrm{d} \mathbf{r}
$$

By substituting (3.1)-(3.3) into (2.3) and equating coefficients, 
one obtains $N$ coupled matrix equations. If the $\rho_{j}^{(n)}$ are known (these can be derived from the molecular Cartesian multipole moments), one can use singular value decomposition to solve for the $\sigma_{j}^{(n)}$. Explicit formulas for the first two Stewart atom moments of various small molecules are given in the Appendix.

Suppose that we have used this approach to find the radial moments of a Stewart atom in a molecule. If all of its moments were known, it would be possible to use these to reconstruct the Stewart atom exactly. However, is a useful extrapolation possible if only, say, $\sigma^{(0)}$ and $\sigma^{(2)}$ are known? This appears to be a seriously underdetermined problem since clearly there are an infinite number of different Stewart atoms possessing these two moments.

We have already noted that Stewart's notion of atoms-inmolecules conceives of these as radially distorted free atoms. Guided by this view, and the observation that free atom densities are approximately piecewise exponential, ${ }^{27}$ we propose that a Stewart atom may be modeled satisfactorily by a linear combination of exponentials. Specifically, first-, second-, and third-row Stewart atoms will be approximated by

$$
\begin{gathered}
\sigma_{\mathrm{H}-\mathrm{He}}(r) \approx Q\left(\frac{\alpha^{3}}{\pi}\right) \mathrm{e}^{-2 \alpha r} \\
\sigma_{\mathrm{Li}-\mathrm{Ne}}(r) \approx 2\left(\frac{c_{1}^{3}}{\pi}\right) \mathrm{e}^{-2 c_{1} r}+Q\left(\frac{\alpha^{5}}{96 \pi}\right) r^{2} \mathrm{e}^{-\alpha r} \\
\sigma_{\mathrm{Na}-\mathrm{Ar}}(r) \approx 2\left(\frac{c_{1}^{3}}{\pi}\right) \mathrm{e}^{-2 c_{1} r}+8\left(\frac{c_{2}^{5}}{96 \pi}\right) r^{2} \mathrm{e}^{-c_{2} r}+ \\
Q\left(\frac{2 \alpha^{7}}{98415 \pi}\right) r^{4} \mathrm{e}^{-2 \alpha r / 3}
\end{gathered}
$$

where the $c_{i}$ are standard Slater exponents ${ }^{28}$ and the amplitude $Q$ and valence exponent $\alpha$ are chosen to reproduce $\sigma^{(0)}$ and $\sigma^{(2)}$. It is easy to show that the parameters in (3.6) are given by

$$
\begin{gathered}
Q=\sigma^{(0)} \\
\alpha=\sqrt{\frac{3 \sigma^{(0)}}{\sigma^{(2)}}}
\end{gathered}
$$

while those in (3.7) are

$$
\begin{gathered}
Q=\sigma^{(0)}-2 \\
\alpha=\sqrt{\frac{30 Q}{\sigma^{(2)}-6 c_{1}^{-2}}}
\end{gathered}
$$

and those in (3.8) are

$$
\begin{gathered}
Q=\sigma^{(0)}-10 \\
\alpha=\sqrt{\frac{126 Q}{\sigma^{(2)}-6 c_{1}^{-2}-240 c_{2}^{-2}}}
\end{gathered}
$$

We will refer to the functions (3.6)-(3.8) as Stewart-Slater

\begin{tabular}{|c|c|c|c|c|c|c|}
\hline \multirow[b]{2}{*}{ atom } & \multicolumn{2}{|c|}{ cation } & \multicolumn{2}{|c|}{ neutral } & \multicolumn{2}{|c|}{ anion } \\
\hline & $\sigma^{(2)}$ & $\alpha$ & $\sigma^{(2)}$ & $\alpha$ & $\sigma^{(2)}$ & $\alpha$ \\
\hline $\mathrm{H}$ & 0.00000 & & 3.00631 & 1.00 & 16.6142 & 0.60 \\
\hline $\mathrm{He}$ & 0.75340 & 2.00 & 2.37633 & 1.59 & 16.4265 & \\
\hline $\mathrm{Li}$ & 0.89167 & & 18.6594 & 1.30 & 85.5732 & 0.84 \\
\hline $\mathrm{Be}$ & 7.28480 & 2.09 & 17.3306 & 1.88 & 63.5252 & 1.19 \\
\hline B & 9.22676 & 2.59 & 15.8156 & 2.41 & 36.1276 & 1.83 \\
\hline $\mathrm{C}$ & 8.11862 & 3.37 & 13.8180 & 2.97 & 25.7429 & 2.42 \\
\hline $\mathrm{N}$ & 7.81729 & 3.95 & 12.1329 & 3.54 & 23.0608 & 2.80 \\
\hline $\mathrm{O}$ & 7.40477 & 4.53 & 11.2578 & 4.02 & 18.3238 & 3.39 \\
\hline $\mathrm{F}$ & 7.16381 & 5.04 & 10.2806 & 4.54 & 15.5376 & 3.94 \\
\hline $\mathrm{Ne}$ & 6.82876 & 5.57 & 9.39476 & 5.07 & 18.5979 & \\
\hline $\mathrm{Na}$ & 6.40361 & & 27.2167 & 2.39 & 100.343 & 1.63 \\
\hline $\mathrm{Mg}$ & 14.0205 & 3.54 & 29.6572 & 3.13 & 106.238 & 1.92 \\
\hline $\mathrm{Al}$ & 17.2968 & 4.21 & 33.1742 & 3.55 & 66.8512 & 2.81 \\
\hline $\mathrm{Si}$ & 20.0798 & 4.64 & 32.1561 & 4.12 & 53.6081 & 3.51 \\
\hline $\mathrm{P}$ & 20.9607 & 5.16 & 30.5152 & 4.71 & 48.8470 & 4.02 \\
\hline S & 20.8119 & 5.75 & 29.3139 & 5.24 & 42.9056 & 4.63 \\
\hline $\mathrm{Cl}$ & 20.7686 & 6.26 & 27.7987 & 5.79 & 38.1730 & 5.24 \\
\hline $\mathrm{Ar}$ & 20.2193 & 6.82 & 26.0643 & 6.38 & 48.7676 & \\
\hline
\end{tabular}
atoms. These "atoms" have a standard Slater core but an outer region with, in general, a nonstandard valence exponent $\alpha$ and a nonintegral number $Q$ of valence electrons.

We note in passing that, when higher Stewart moments are available, one can construct "double-split-valence" StewartSlater atoms. These are analogous to (3.6)-(3.8) but have two valence amplitudes and two valence exponents chosen to
TABLE 1: Second Radial Moments and Stewart Exponents in Atoms and Their Ions ${ }^{a}$

${ }^{a}$ From UHF/6-311++G $(d, p)$ electron densities.

reproduce $\sigma^{(0)}, \sigma^{(2)}, \sigma^{(4)}$, and $\sigma^{(6)}$. We have not examined these more accurate approximate Stewart atoms in the present paper.

The Stewart-Slater decomposition provides a convenient, semiquantitative route to the electrostatic potential in and around a molecule. This is of central importance for semiclassical modeling in many different contexts. The potentials of Slatertype density functions are given by the simple expressions

$$
\begin{gathered}
V\left[\left(\frac{\beta^{3}}{8 \pi}\right) \mathrm{e}^{-\beta r}\right]=\frac{1}{r}-\frac{\mathrm{e}^{-z}}{r}\left(1+\frac{z}{2}\right) \\
V\left[\left(\frac{\beta^{5}}{96 \pi}\right) r^{2} \mathrm{e}^{-\beta r}\right]=\frac{1}{r}-\frac{\mathrm{e}^{-z}}{r}\left(1+\frac{3 z}{4}+\frac{z^{2}}{4}+\frac{z^{3}}{24}\right)
\end{gathered}
$$

$$
\begin{aligned}
V\left[\left(\frac{\beta^{7}}{2880 \pi}\right) r^{4} \mathrm{e}^{-\beta r}\right] & = \\
& \frac{1}{r}-\frac{\mathrm{e}^{-z}}{r}\left(1+\frac{5 z}{6}+\frac{z^{2}}{3}+\frac{z^{3}}{12}+\frac{z^{4}}{72}+\frac{z^{5}}{720}\right)
\end{aligned}
$$

where $z=\beta r$. We will report results using this approach elsewhere.

\section{Results and Discussion}

We do not seek in this paper to present an exhaustive numerical study of Stewart atoms. Rather, we have selected a sample of mainly organic molecules and studied these carefully in an attempt to learn something about the typical behavior of Stewart and Stewart-Slater atoms. All of the data discussed below are based on unrestricted Hartree-Fock (UHF) densities. This level suffices for our present purposes for it is well-known that the effects of correlation on molecular densities are generally quite small. Moreover, the basis set that we have employed, 6-311++ $\mathrm{G}(d, p)$, is fairly large and well balanced and yields results quite close to the HF limit. All calculations were performed using the Q-CHEM program ${ }^{25}$ on an IBM 43P workstation.

To begin, we calculated the first two radial moments of the density of the first 18 atoms and their cations and anions. These are listed in Table 1 with the Stewart exponent $\alpha$ of the corresponding Stewart-Slater atoms. The exponents, which turn out to be slightly smaller than the standard valence Slater values, ${ }^{28}$ are a useful reference set against which to compare the exponents of Stewart atoms in molecules. 
TABLE 2: Radial Moments about Nuclei in Hydrides ${ }^{a}$

\begin{tabular}{|c|c|c|c|c|c|c|}
\hline molecule & & $\rho^{(0)}$ & $\rho^{(2)}$ & $\rho^{(4)}$ & $\rho^{(6)}$ & $\rho^{(8)}$ \\
\hline $\mathrm{H}_{2}$ & $\mathrm{H}$ & 2.00000 & 6.12247 & 40.7147 & 473.765 & 8395.74 \\
\hline \multirow[t]{2}{*}{$\mathrm{LiH}$} & $\mathrm{Li}$ & 4.00000 & 26.9052 & 510.025 & 15763.8 & 1127890 \\
\hline & $\mathrm{H}$ & 4.00000 & 31.1758 & 378.068 & 7770.90 & 557740 \\
\hline \multirow[t]{2}{*}{$\mathrm{BeH}_{2}$} & $\mathrm{Be}$ & 6.00000 & 31.6082 & 383.503 & 7088.32 & 191800 \\
\hline & $\mathrm{H}$ & 6.00000 & 69.6748 & 1579.54 & 50563.9 & 2032730 \\
\hline \multirow[t]{2}{*}{$\mathrm{BH}_{3}$} & $\mathrm{~B}$ & 8.00000 & 34.5908 & 330.535 & 4895.63 & 105269 \\
\hline & $\mathrm{H}$ & 8.00000 & 75.1215 & 1183.36 & 25923.1 & 737344 \\
\hline \multirow[t]{2}{*}{$\mathrm{CH}_{4}$} & $\mathrm{C}$ & 10.0000 & 35.7759 & 285.267 & 3712.88 & 78381.9 \\
\hline & $\mathrm{H}$ & 10.0000 & 78.1777 & 970.711 & 16981.1 & 406530 \\
\hline \multirow[t]{2}{*}{$\mathrm{NH}_{3}$} & $\mathrm{~N}$ & 10.0000 & 26.9878 & 185.483 & 2330.11 & 52842.1 \\
\hline & $\mathrm{H}$ & 10.0000 & 61.7379 & 613.345 & 9163.29 & 206468 \\
\hline \multirow[t]{2}{*}{$\mathrm{OH}_{2}$} & $\mathrm{O}$ & 10.0000 & 19.7687 & 108.946 & 1114.69 & 20275.5 \\
\hline & $\mathrm{H}$ & 10.0000 & 50.1919 & 393.869 & 4683.02 & 83965.1 \\
\hline \multirow[t]{2}{*}{$\mathrm{FH}$} & $\mathrm{F}$ & 10.0000 & 13.9224 & 57.8241 & 454.895 & 6265.01 \\
\hline & $\mathrm{H}$ & 10.0000 & 41.7578 & 255.447 & 2288.34 & 30103.5 \\
\hline \multirow[t]{2}{*}{$\mathrm{NaH}$} & $\mathrm{Na}$ & 12.0000 & 40.3036 & 826.126 & 29411.8 & 2235450 \\
\hline & $\mathrm{H}$ & 12.0000 & 151.195 & 2283.85 & 42767.7 & 1818130 \\
\hline \multirow[t]{2}{*}{$\mathrm{MgH}_{2}$} & $\mathrm{Mg}$ & 14.0000 & 54.6537 & 896.200 & 22328.5 & 790150 \\
\hline & $\mathrm{H}$ & 14.0000 & 202.817 & 5278.10 & 223720 & 12716600 \\
\hline \multirow[t]{2}{*}{$\mathrm{AlH}_{3}$} & $\mathrm{Al}$ & 16.0000 & 63.7955 & 887.964 & 18723.3 & 594013 \\
\hline & $\mathrm{H}$ & 16.0000 & 208.062 & 4335.69 & 133852 & 5561330 \\
\hline \multirow[t]{2}{*}{$\mathrm{SiH}_{4}$} & $\mathrm{Si}$ & 18.0000 & 69.0237 & 824.608 & 14745.7 & 376062 \\
\hline & $\mathrm{H}$ & 18.0000 & 210.367 & 3741.17 & 94638.7 & 3169720 \\
\hline \multirow[t]{2}{*}{$\mathrm{PH}_{3}$} & $\mathrm{P}$ & 18.0000 & 56.5346 & 603.288 & 10224.1 & 258967 \\
\hline & $\mathrm{H}$ & 18.0000 & 174.048 & 2476.11 & 51872.2 & 1551750 \\
\hline \multirow{2}{*}{$\mathrm{SH}_{2}$} & $\mathrm{~S}$ & 18.0000 & 44.6225 & 403.613 & 6156.84 & 145233 \\
\hline & $\mathrm{H}$ & 18.0000 & 149.756 & 1804.93 & 32402.0 & 853419 \\
\hline \multirow[t]{2}{*}{$\mathrm{ClH}$} & $\mathrm{Cl}$ & 18.0000 & 34.4393 & 252.336 & 3245.95 & 68254.7 \\
\hline & $\mathrm{H}$ & 18.0000 & 130.790 & 1334.45 & 20009.5 & 441817 \\
\hline
\end{tabular}

${ }^{a}$ From HF/6-311++G(d,p)//MP2/6-31G $(d)$ electron densities.

The radial moments $\rho^{(n)}$ of the first-row and second-row hydrides are listed in Table 2 . We have then used these, and formulas A.8-A.13, to calculate $\sigma^{(0)}$ and $\sigma^{(2)}$ for each of the Stewart atoms in these molecules. These moments, along with Stewart charges and exponents from (3.9)-(3.14) and the Mulliken charges, are given in Table 3. Using a singular value decomposition to solve for the $\sigma^{(n)}$, we have also obtained the Stewart moments, charges, and exponents and Mulliken charges of a number of other molecules and these are listed in Table 4.

The Stewart charges in Table 3 yield simple but important insights into the nature of the bonding in the molecules considered. Moving along a row of the Periodic Table from the alkali hydrides to the hydrogen halides, the $\mathrm{H}$ atom charge changes from negative $(-0.78$ in $\mathrm{LiH})$ to positive $(+0.47$ in $\mathrm{HF}$ ), as one would expect. Both the Stewart and Mulliken charges on $\mathrm{H}$ are shown in Figure 1 and, while the two schemes show similar gross trends, it is clear that they differ substantially in detail. Apart from $\mathrm{SiH}_{4}$ and $\mathrm{PH}_{3}$, the Stewart charges are consistently larger than the Mulliken counterparts. The hydride with the least polar bonds is $\mathrm{PH}_{3}$ (Stewart) or $\mathrm{H}_{2} \mathrm{~S}$ (Mulliken): standard Pauling electronegativity values ${ }^{29}$ support the Stewart choice.

Because the Stewart exponent $\alpha$ is related to the radial extent of the Stewart atom, it may be used to deduce the effects of bonding on the sizes of atoms. For example, the increase in $\alpha$ from 1.00 in the free $\mathrm{H}$ atom to 1.20 in $\mathrm{H}_{2}$ illustrates the contraction that accompanies bond formation, a phenomenon that has been recognized since the early days of molecular quantum mechanics ${ }^{30}$ and which has been cited ${ }^{31}$ as the driving force for bonding in the $\mathrm{H}_{2}{ }^{+}$molecule. We observe an analogous effect in the isoelectronic species $\mathrm{Li}_{2}$ but, in contrast, no contraction is observed in $\mathrm{N}_{2}$ and, indeed, a very slight expansion is found in $\mathrm{F}_{2}$.

The heavy atom exponents in the metal hydrides are much greater than in the free atoms suggesting compact quasi-cations. Conversely, the $\mathrm{H}$ exponents in $\mathrm{LiH}$ and $\mathrm{NaH}$ are smaller than
TABLE 3: Stewart Moments, Stewart Parameters, and Mulliken Charges in Hydrides ${ }^{a}$

\begin{tabular}{|c|c|c|c|c|c|c|}
\hline \multirow[b]{2}{*}{ molecule } & & \multicolumn{2}{|c|}{$\begin{array}{l}\text { Stewart } \\
\text { moments }\end{array}$} & \multicolumn{2}{|c|}{$\begin{array}{c}\text { Stewart } \\
\text { parameters }\end{array}$} & \multirow{2}{*}{$\begin{array}{c}\text { Mulliken } \\
\text { charge }\end{array}$} \\
\hline & & $\sigma^{(0)}$ & $\sigma^{(2)}$ & charge & $\alpha$ & \\
\hline $\mathrm{H}_{2}$ & $\mathrm{H}$ & 1.00000 & 2.09095 & 0.000 & 1.20 & 0.000 \\
\hline \multirow[t]{2}{*}{$\mathrm{LiH}$} & $\mathrm{Li}$ & 2.22243 & 2.21805 & +0.778 & 2.19 & +0.420 \\
\hline & $\mathrm{H}$ & 1.77757 & 7.62303 & -0.778 & 0.84 & -0.420 \\
\hline \multirow[t]{2}{*}{$\mathrm{BeH}_{2}$} & $\mathrm{Be}$ & 3.33485 & 7.89079 & +0.665 & 2.32 & +0.333 \\
\hline & $\mathrm{H}$ & 1.33258 & 3.40426 & -0.333 & 1.08 & -0.167 \\
\hline \multirow[t]{2}{*}{$\mathrm{BH}_{3}$} & B & 4.29968 & 6.76554 & +0.700 & 3.26 & +0.211 \\
\hline & $\mathrm{H}$ & 1.23344 & 3.02606 & -0.233 & 1.11 & -0.070 \\
\hline \multirow[t]{2}{*}{$\mathrm{CH}_{4}$} & $\mathrm{C}$ & 6.57828 & 14.3215 & -0.578 & 3.12 & -0.469 \\
\hline & $\mathrm{H}$ & 0.85543 & 1.73644 & +0.145 & 1.22 & +0.117 \\
\hline \multirow[t]{2}{*}{$\mathrm{NH}_{3}$} & $\mathrm{~N}$ & 7.98486 & 15.5570 & -0.985 & 3.41 & -0.675 \\
\hline & $\mathrm{H}$ & 0.67171 & 1.33030 & +0.328 & 1.23 & +0.225 \\
\hline \multirow[t]{2}{*}{$\mathrm{OH}_{2}$} & $\mathrm{O}$ & 8.78853 & 13.6470 & -0.789 & 3.88 & -0.512 \\
\hline & $\mathrm{H}$ & 0.60573 & 1.03157 & +0.394 & 1.33 & +0.256 \\
\hline \multirow[t]{2}{*}{ FH } & $\mathrm{F}$ & 9.46865 & 11.4763 & -0.469 & 4.43 & -0.308 \\
\hline & $\mathrm{H}$ & 0.53135 & 0.79121 & +0.469 & 1.42 & +0.308 \\
\hline \multirow[t]{2}{*}{$\mathrm{NaH}$} & $\mathrm{Na}$ & 10.2232 & 8.50973 & +0.777 & 2.90 & +0.503 \\
\hline & $\mathrm{H}$ & 1.77676 & 8.46723 & -0.777 & 0.79 & -0.503 \\
\hline \multirow[t]{2}{*}{$\mathrm{MgH}_{2}$} & $\mathrm{Mg}$ & 11.0340 & 14.0301 & +0.966 & 3.59 & +0.603 \\
\hline & $\mathrm{H}$ & 1.48301 & 4.61703 & -0.483 & 0.98 & -0.301 \\
\hline \multirow[t]{2}{*}{$\mathrm{AlH}_{3}$} & $\mathrm{Al}$ & 11.7653 & 12.8552 & +1.235 & 4.78 & +0.863 \\
\hline & $\mathrm{H}$ & 1.41155 & 4.25259 & -0.412 & 1.00 & -0.288 \\
\hline \multirow[t]{2}{*}{$\mathrm{SiH}_{4}$} & $\mathrm{Si}$ & 13.4219 & 21.8873 & +0.578 & 4.72 & +0.799 \\
\hline & $\mathrm{H}$ & 1.14453 & 2.79682 & -0.145 & 1.11 & -0.200 \\
\hline \multirow[t]{2}{*}{$\mathrm{PH}_{3}$} & $\mathrm{P}$ & 15.2238 & 30.8427 & -0.224 & 4.78 & +0.244 \\
\hline & $\mathrm{H}$ & 0.92539 & 1.95264 & +0.075 & 1.19 & -0.081 \\
\hline \multirow[t]{2}{*}{$\mathrm{SH}_{2}$} & $S$ & 16.3109 & 30.0198 & -0.311 & 5.30 & -0.064 \\
\hline & $\mathrm{H}$ & 0.84455 & 1.89027 & +0.155 & 1.16 & +0.032 \\
\hline \multirow[t]{2}{*}{$\mathrm{ClH}$} & $\mathrm{Cl}$ & 17.2342 & 28.2703 & -0.234 & 5.83 & -0.133 \\
\hline & $\mathrm{H}$ & 0.76580 & 1.68859 & +0.234 & 1.17 & +0.133 \\
\hline
\end{tabular}

${ }^{a}$ From HF/6-311++G(d,p)//MP2/6-31G $(d)$ electron densities.

unity and attest to the diffuseness of the quasi-anionic hydride moiety. In general, the $\mathrm{H}$ exponents in the hydrides studied can be rationalized by two propositions: (a) when the atom becomes bonded, it tends to contract; (b) if the atom becomes positively (negatively) charged by bonding, it tends to contract 
TABLE 4: Stewart Moments, Stewart Parameters, and Mulliken Charges in Other Molecules ${ }^{a}$

\begin{tabular}{|c|c|c|c|c|c|c|}
\hline \multirow{2}{*}{ molecule } & & \multicolumn{2}{|c|}{ Stewart moments } & \multicolumn{2}{|c|}{ Stewart parameters } & \multirow{2}{*}{$\begin{array}{c}\text { Mulliken } \\
\text { charge }\end{array}$} \\
\hline & & $\sigma^{(0)}$ & $\sigma^{(2)}$ & charge & $\alpha$ & \\
\hline $\mathrm{Li}_{2}$ & $\mathrm{Li}$ & 00000 & 13.3 & & 1.55 & 0.000 \\
\hline $\mathrm{N}_{2}$ & $\mathrm{~N}$ & 00000 & & & 25 & .000 \\
\hline $\mathrm{F}_{2}$ & $\mathrm{~F}$ & 9.00000 & 10.4340 & 0.000 & 4.50 & 0.000 \\
\hline \multirow[t]{2}{*}{$\mathrm{LiF}$} & $\mathrm{Li}$ & 2.12192 & 1.31726 & +0.878 & 2.72 & -0.689 \\
\hline & $\mathrm{F}$ & 7808 & 13. & -0.8 & .18 & -0.689 \\
\hline \multirow[t]{2}{*}{$\mathrm{CO}$} & $\mathrm{C}$ & 5732 & 13 & +0. & .96 & +0.090 \\
\hline & $\mathrm{O}$ & 4268 & 10. & -0 & 4.14 & -0.090 \\
\hline \multirow[t]{2}{*}{$\mathrm{CO}_{2}$} & $\mathrm{C}$ & 5.10444 & 8.68 & +0.896 & 3.31 & +0.646 \\
\hline & $\mathrm{O}$ & 8.44778 & 12.0374 & -0.448 & 4.03 & -0.323 \\
\hline \multirow[t]{3}{*}{$\mathrm{CH}_{3} \mathrm{~F}$} & $\mathrm{C}$ & 5.87424 & 11.6 & +0 . & 3.19 & -0.137 \\
\hline & $\mathrm{H}$ & & & +0 & 1.33 & +0.124 \\
\hline & $\mathrm{F}$ & & & -0 . & 2 & -0 \\
\hline \multirow[t]{3}{*}{$\mathrm{CH}_{2} \mathrm{~F}_{2}$} & $\mathrm{C}$ & 5 & 11. & +0 & 3.12 & +0.160 \\
\hline & $\mathrm{H}$ & & & +0 . & 1 & +0.137 \\
\hline & $\mathrm{F}$ & 9.27259 & 10.89 & -0.2 & 4.49 & -0.217 \\
\hline \multirow[t]{3}{*}{$\mathrm{CHF}_{3}$} & $\mathrm{C}$ & 5.33875 & 11.3414 & +0.661 & 3.00 & +0.422 \\
\hline & $\mathrm{H}$ & 0.93866 & & +0.061 & 1.68 & +0.19 \\
\hline & $\mathrm{F}$ & & & -0 & 4.56 & -0.20 \\
\hline \multirow[t]{2}{*}{$\mathrm{C}_{2} \mathrm{H}_{2}$} & $\mathrm{C}$ & 6. & 14. & -0 . & 2.98 & -0.21 \\
\hline & $\mathrm{H}$ & & & +0 . & 1.5 & \\
\hline \multirow[t]{2}{*}{$\mathrm{C}_{2} \mathrm{H}_{4}$} & $\mathrm{C}$ & 6.39744 & 15.76 & -0 . & 2.91 & -0.22 \\
\hline & $\mathrm{H}$ & & 1. & +0 . & 1.53 & +0.11 \\
\hline \multirow[t]{2}{*}{$\mathrm{C}_{2} \mathrm{H}_{6}$} & $\mathrm{C}$ & 5.69473 & 4.76645 & +0.305 & 4.92 & -0.34 \\
\hline & $\mathrm{H}$ & 1.10176 & 3.41 & -0.102 & 0.98 & +0.11 \\
\hline \multirow[t]{2}{*}{$\mathrm{C}_{3} \mathrm{H}_{3}^{+}$} & $\mathrm{C}$ & 5.88740 & 10.2853 & +0.113 & 3.40 & +0.033 \\
\hline & $\mathrm{H}$ & 0.77927 & 1.41283 & +0.221 & 1.29 & +0.30 \\
\hline \multirow[t]{2}{*}{$\mathrm{C}_{6} \mathrm{H}_{6}$} & $\mathrm{C}$ & 6.15405 & 14.3378 & -0.154 & 2.97 & -0.19 \\
\hline & $\mathrm{H}$ & 0.84595 & 0.94835 & +0.154 & 1.64 & +0.19 \\
\hline
\end{tabular}

${ }^{a}$ From HF/6-311++G $(d, p) / / \mathrm{HF} / 6-31 \mathrm{G}(d)$ electron densities.

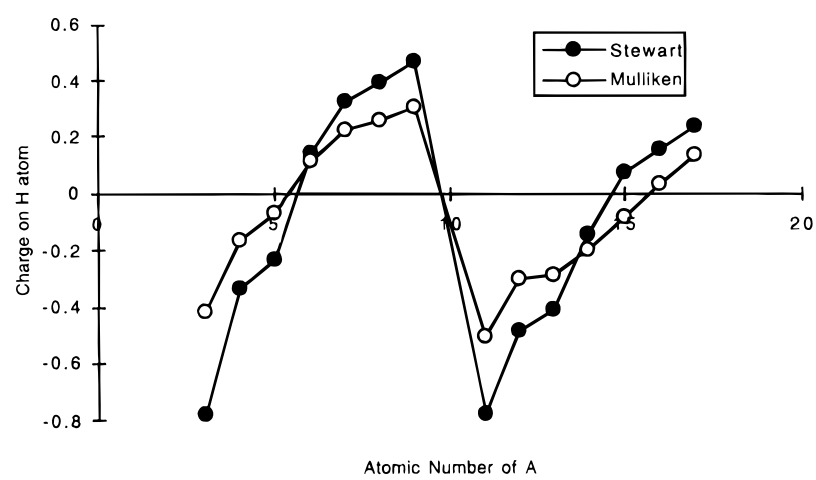

Figure 1. $\mathrm{H}$ atom charge in $\mathrm{AH}_{n}$ (values from Table 3).

(expand). The net effect of bonding on the atom's size is the sum of these two, possibly opposing, effects and we note in passing that $\mathrm{AlH}_{3}$ appears to be a system in which almost perfect cancellation occurs.

The LiF molecule exemplifies highly ionic bonding and is commonly represented $\mathrm{Li}^{+} \mathrm{F}^{-}$but its Mulliken charges $( \pm 0.69)$ imply much less polarity than do the Stewart values $( \pm 0.88)$. The latter, it should be noted, reproduce the dipole moment of the electron density exactly. The $\mathrm{Li}$ exponent is more than double that of the free $\mathrm{Li}$ atom and, indeed, the $\sigma_{\mathrm{Li}}^{(2)}$ value in Table 4 is not much greater than that of the $\mathrm{Li}^{+}$cation. Likewise, the $\mathrm{F}$ exponent is closer to that of the $\mathrm{F}^{-}$anion than the free $\mathrm{F}$ atom.

The dipole moment of the $\mathrm{CO}$ molecule is small and its direction (the $\mathrm{O}$ atom is positive) is predicted incorrectly by Hartree-Fock theory but this is of no consequence for our purposes. We find that the Mulliken charges are twice as large as the Stewart ones but that both are very small. The $\mathrm{C}$ and $\mathrm{O}$ Stewart atoms are both similar in size to free atoms. The Stewart analysis of the $\mathrm{CO}_{2}$ molecule, however, is strikingly different yielding highly charged atoms. Indeed, the charge and exponent of the Stewart $\mathrm{C}$ atom are very close to those of a $\mathrm{C}^{+}$ cation.

Stewart has previously calculated ${ }^{23}$ the charges of the fluoromethanes $\mathrm{CH}_{n} \mathrm{~F}_{4-n}$ and we have included results for $\mathrm{CH}_{3} \mathrm{~F}$, $\mathrm{CH}_{2} \mathrm{~F}_{2}$, and $\mathrm{CHF}_{3}$ in Table 4. The charge distribution in these systems is of interest and we find that the Stewart and Mulliken analyses afford rather different descriptions. While agreeing that the $\mathrm{F}$ atoms bear significant negative charges that diminish slightly as the degree of substitution increases, they disagree in their allocation of the resulting positive charge. More specifically, while the Stewart analysis allots almost no charge at all to the $\mathrm{H}$ atoms and leaves the $\mathrm{C}$ atom to shoulder this burden alone, the Mulliken analysis is more equitable and uses the $\mathrm{H}$ atoms to reduce the $\mathrm{C}$ charge by roughly $1 / 4$. In $\mathrm{CH}_{3} \mathrm{~F}$, however, the Mulliken scheme leads to the counterintuitive proposition that the $\mathrm{C}$ atom is negatively charged.

Table 4 concludes with systems containing a variety of $\mathrm{C}-\mathrm{C}$ bond types: single, double, triple, and aromatic. The charges on the $\mathrm{C}$ atoms are surprisingly variable considering that these molecules are all hydrocarbons. The Mulliken and Stewart schemes concur that the alkene, alkyne, and benzene $\mathrm{C}$ atoms bear negative charges but the Stewart charges are larger than the Mulliken ones in $\mathrm{C}_{2} \mathrm{H}_{2}$ and $\mathrm{C}_{2} \mathrm{H}_{4}$ and smaller in $\mathrm{C}_{6} \mathrm{H}_{6}$. The $\mathrm{C}$ atoms in all three compounds are found by Stewart analysis to be similar in size to free $\mathrm{C}$ atoms whereas the $\mathrm{H}$ atoms, which bear positive charges, are found to be much smaller than their free counterparts. The simplest aromatic molecule is the cyclopropenium cation $\mathrm{C}_{3} \mathrm{H}_{3}{ }^{+}$and it is intriguing to find that, whereas Mulliken analysis puts almost all of the excess charge on the $\mathrm{H}$ atoms, Stewart analysis places a third of it on the ring and yields $\mathrm{C}$ atoms that are only as large as $\mathrm{C}^{+}$cations.

The Stewart results for $\mathrm{C}_{2} \mathrm{H}_{6}$ contain a cautionary message. Because the $\mathrm{C}$ atoms in this molecule are "buried" within a shell of $\mathrm{H}$ atoms, they are relatively unimportant for modeling the molecular density. Stewart analysis therefore allots relatively little density to them and, as a consequence, they are positively charged. Moreover, the resulting Stewart exponent is anomalously large indicating, at first glance, an extremely compact atom. The true explanation, however, for the surprisingly small second moment of the $\mathrm{C}$ atom is that $\sigma_{\mathrm{C}}(r)$ is not strictly positive. This is confirmed by its fourth moment which we find to be negative. We conjecture that it may not be uncommon for the Stewart atoms of "buried" nuclei to contain nodes.

The last stage in this preliminary investigation is to examine the effect of basis set on Stewart atoms. It is well-known that Mulliken analysis is very sensitive to the basis used and that, in order to obtain meaningful charges, it is important that the set used be "well balanced". To study the sensitivity of the Stewart charges and exponents, we arbitrarily selected the $\mathrm{CH}_{3} \mathrm{~F}$ molecule and performed a Stewart analysis on its Hartree-Fock density determined with a range of basis sets from STO-3G to $6-311++\mathrm{G}(3 d f, 3 p d)$. The results are summarized in Table 5 and it is clear from these that the charges are strongly basis set dependent but that the exponents are much less so. The instability of the Stewart charges is a direct reflection of the instability of the molecular dipole moment with respect to basis set improvements.

\section{Concluding Remarks}

We have shown that Stewart's scheme for partitioning molecular electron densities yields "atoms" that are intuitively plausible and chemically useful. By augmenting his approach with the additional approximation that the valence part of a 
TABLE 5: Dipole Moment and Stewart Parameters in the $\mathrm{CH}_{3} \mathrm{~F}$ Molecule ${ }^{a}$

\begin{tabular}{|c|c|c|c|c|c|c|c|}
\hline \multirow[b]{2}{*}{ basis set } & \multirow{2}{*}{$\begin{array}{l}\text { dipole } \\
\text { moment }\end{array}$} & \multicolumn{2}{|c|}{$\mathrm{C}$ parameters } & \multicolumn{2}{|c|}{$\mathrm{H}$ parameters } & \multicolumn{2}{|c|}{ F parameters } \\
\hline & & charge & $\alpha$ & ch & $\alpha$ & Sit & $\alpha$ \\
\hline $3 \mathrm{C}$ & & & & & 1 & & 5 \\
\hline & & 10.2 & 3.09 & +0 & 1.40 & & 4.61 \\
\hline & 22 & +0 & 3.17 & +0 . & 1.37 & 54 & 4.48 \\
\hline & 2.4 & + & 3.16 & +0 & 1.39 & -0 & 4.45 \\
\hline$(d)$ & 4 & +0 & 3.14 & +0 & 1.36 & -0 & 4.55 \\
\hline$(d)$ & 2 & 93 & 3.13 & +0 & 1.38 & -( & 4.50 \\
\hline ( & 2.0 & +0.093 & 3.15 & +0 . & 1.37 & -0.269 & 4.50 \\
\hline$+\mathrm{G}(d, p)$ & 2.1 & +0.121 & 3.17 & +0 & 1.34 & -0 & 4.42 \\
\hline $1++\mathrm{G}(d, p)$ & 2.1 & +0.126 & 3.19 & +0.055 & 1.33 & -0.285 & 4.42 \\
\hline$-311++\mathrm{G}(2 d, 2 p)$ & 2.0 & +0.045 & 3.16 & +0.071 & 1.33 & -0.254 & 4.44 \\
\hline $1++\mathrm{G}(3 d, 3 p)$ & 1.9 & +0.016 & 3.14 & +0.077 & 1.33 & -0.242 & 4.45 \\
\hline $6-311++\mathrm{G}(3 d f, 3 p d)$ & 1.9800 & +0.034 & 3.15 & +0.072 & 1.33 & -0.245 & 4.45 \\
\hline
\end{tabular}

${ }^{a}$ From Hartree-Fock densities at the HF/6-31G $(d)$ geometry.

Stewart atom is a Slater exponential, we have demonstrated that the calculations can be automated and run as an inexpensive population analysis tool in a quantum chemistry package. We propose that a sum of Stewart-Slater atoms may constitute a useful and computationally efficient approximate molecular density for use in a variety of contexts. We will report further on such applications in a subsequent paper.

Acknowledgment. This research was sparked by a spirited argument with Prof. Bob Stewart (Carnegie Mellon), Dr. Benny Johnson (Q-CHEM Inc.), and Prof. John Pople (Northwestern) and was supported in part by a grant from the Massey University Research Fund. We thank Dr. Terry Adams for his useful comments on this manuscript and Prof. Stewart for kindly supplying ref 23 .

\section{Appendix: Stewart Moments in Various Molecules}

Applying the theory of section 3 to a diatomic $\mathrm{AB}$ yields the coupled matrix equation

$$
\begin{aligned}
& {\left[\begin{array}{ll}
\mathbf{1} & \mathbf{T} \\
\mathbf{T} & \mathbf{1}
\end{array}\right]\left[\begin{array}{l}
\sigma_{\mathrm{A}} \\
\sigma_{\mathrm{B}}
\end{array}\right]=\left[\begin{array}{l}
\rho_{\mathrm{A}} \\
\rho_{\mathrm{B}}
\end{array}\right]} \\
& \mathbf{T}=\left[\begin{array}{llllll}
1 & & & & & \\
R^{2} & 1 & & & & \\
R^{4} & 10 /{ }_{3} R^{2} & 1 & & & \\
R^{6} & 7 R^{4} & 7 R^{2} & 1 & & \\
R^{8} & 12 R^{6} & 126 / R_{5} & 12 R^{2} & 1 & \\
\vdots & \vdots & \vdots & \vdots & \vdots & \ddots
\end{array}\right]
\end{aligned}
$$

In the case of a homonuclear diatomic $\mathrm{A}_{2}$, symmetry reduces (A.1) to

$$
(\mathbf{1}+\mathbf{T}) \sigma=\rho
$$

and the invertibility of the $(\mathbf{1}+\mathbf{T})$ matrix quickly yields

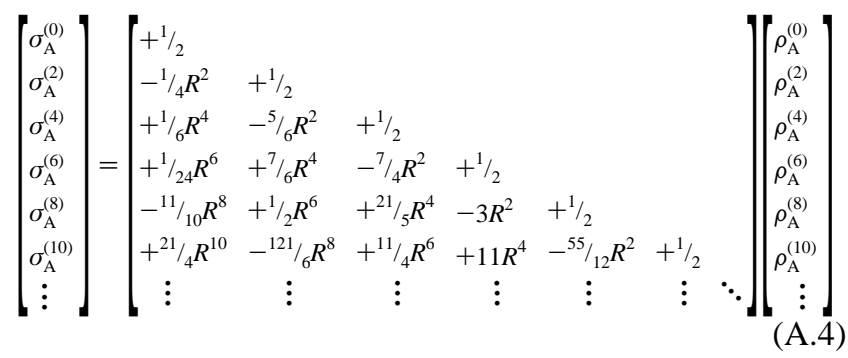

In the case of a general heteronuclear diatomic, (A.1) has the formal solution

$$
\left[\begin{array}{l}
\sigma_{\mathrm{A}} \\
\sigma_{\mathrm{B}}
\end{array}\right]=\left(\mathbf{1}-\mathbf{T}^{2}\right)^{-1}\left[\begin{array}{rr}
\mathbf{1} & -\mathbf{T} \\
-\mathbf{T} & \mathbf{1}
\end{array}\right]\left[\begin{array}{l}
\rho_{\mathrm{A}} \\
\rho_{\mathrm{B}}
\end{array}\right]
$$

but this expression seems problematic because $\left(\mathbf{1}-\mathbf{T}^{2}\right)$ does not possess a well-defined inverse. Analogous problems are observed for any polyatomic and reflect the fact that the elements of $\rho_{1}$ and $\rho_{2}$ are not all independent. In particular, we have $\rho_{\mathrm{A}}^{(0)}=\rho_{\mathrm{B}}^{(0)}$. To avoid the singularity in $\left(\mathbf{1}-\mathbf{T}^{2}\right)$, however, one can employ a pseudoinverse such as

$$
\begin{aligned}
& \left(\mathbf{1}-\mathbf{T}^{2}\right)^{-1}=
\end{aligned}
$$

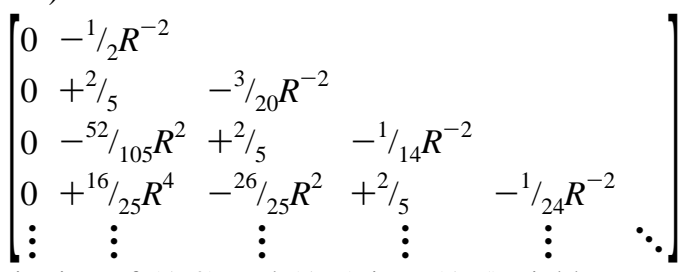

Substitution of (A.2) and (A.6) into (A.5) yields

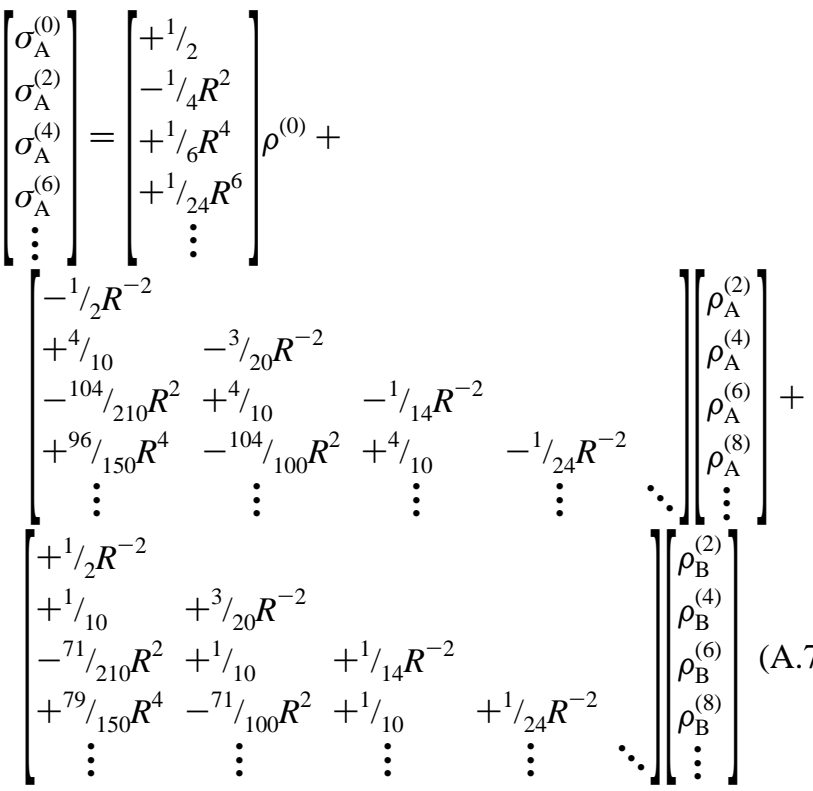

In this way, we have obtained formulas for the following stoichiometries and symmetries:

$\mathbf{A B}$ (point group $=C_{\infty v}$ )

$$
\sigma_{\mathrm{A}}^{(0)}=\frac{\rho^{(0)}}{2}-\frac{\rho_{\mathrm{A}}^{(2)}-\rho_{\mathrm{B}}^{(2)}}{2 R^{2}}
$$

$$
\sigma_{\mathrm{A}}^{(2)}=-\frac{R^{2} \rho^{(0)}}{4}+\frac{4 \rho_{\mathrm{A}}^{(2)}+\rho_{\mathrm{B}}^{(2)}}{10}-\frac{3 \rho_{\mathrm{A}}^{(4)}-3 \rho_{\mathrm{B}}^{(4)}}{20 R^{2}}
$$

$\mathbf{A B}_{2}$ (point group $\left.=D_{\infty h}\right)$

$$
\begin{gathered}
\sigma_{\mathrm{A}}^{(0)}=\frac{11 \rho^{(0)}}{8}+\frac{5 \rho_{\mathrm{A}}^{(2)}}{4 R^{2}}+\frac{3 \rho_{\mathrm{A}}^{(4)}-3 \rho_{\mathrm{B}}^{(4)}}{8 R^{4}} \\
\sigma_{\mathrm{B}}^{(0)}=-\frac{3 \rho^{(0)}}{16}-\frac{5 \rho_{\mathrm{A}}^{(2)}}{8 R^{2}}-\frac{3 \rho_{\mathrm{A}}^{(4)}-3 \rho_{\mathrm{B}}^{(4)}}{16 R^{4}}
\end{gathered}
$$

$\sigma_{\mathrm{A}}^{(2)}=\frac{3 R^{2} \rho^{(0)}}{28}+\frac{87 \rho_{\mathrm{A}}^{(2)}}{56}+\frac{24 \rho_{\mathrm{A}}^{(4)}-3 \rho_{\mathrm{B}}^{(4)}}{56 R^{2}}+\frac{3 \rho_{\mathrm{A}}^{(6)}-3 \rho_{\mathrm{B}}^{(6)}}{56 R^{4}}$ 


$$
\sigma_{\mathrm{B}}^{(2)}=\frac{15 R^{2} \rho^{(0)}}{112}+\frac{39 \rho_{\mathrm{A}}^{(2)}}{112}-\frac{3 \rho_{\mathrm{A}}^{(4)}+18 \rho_{\mathrm{B}}^{(4)}}{112 R^{2}}-\frac{3 \rho_{\mathrm{A}}^{(6)}-3 \rho_{\mathrm{B}}^{(6)}}{112 R^{4}}
$$

$\mathbf{A B}_{3}$ (point group $=D_{3 h}$ )

$$
\begin{gathered}
\sigma_{\mathrm{A}}^{(0)}=\frac{5 \rho^{(0)}}{2}+\frac{5 \rho_{\mathrm{A}}^{(2)}}{R^{2}}+\frac{3 \rho_{\mathrm{A}}^{(4)}-3 \rho_{\mathrm{B}}^{(4)}}{2 R^{4}} \\
\sigma_{\mathrm{B}}^{(0)}=-\frac{\rho^{(0)}}{2}-\frac{5 \rho_{\mathrm{A}}^{(2)}}{3 R^{2}}-\frac{\rho_{\mathrm{A}}^{(4)}-\rho_{\mathrm{B}}^{(4)}}{2 R^{4}} \\
\sigma_{\mathrm{A}}^{(2)}=\frac{15 R^{2} \rho^{(0)}}{14}+\frac{75 \rho_{\mathrm{A}}^{(2)}}{14}+\frac{33 \rho_{\mathrm{A}}^{(4)}-12 \rho_{\mathrm{B}}^{(4)}}{14 R^{2}}+\frac{3 \rho_{\mathrm{A}}^{(6)}-3 \rho_{\mathrm{B}}^{(6)}}{14 R^{4}} \\
\sigma_{\mathrm{B}}^{(2)}=\frac{R^{2} \rho^{(0)}}{7}+\frac{3 \rho_{\mathrm{A}}^{(2)}}{14}-\frac{4 \rho_{\mathrm{A}}^{(4)}+3 \rho_{\mathrm{B}}^{(4)}}{14 R^{2}}-\frac{\rho_{\mathrm{A}}^{(6)}-\rho_{\mathrm{B}}^{(6)}}{14 R^{4}}
\end{gathered}
$$

$\mathbf{A B}_{4}$ (point group $=T_{d}$ )

$$
\begin{gathered}
\sigma_{\mathrm{A}}^{(0)}=\frac{7 \rho^{(0)}}{16}-\frac{63 \rho_{\mathrm{A}}^{(2)}}{16 R^{2}}-\frac{63 \rho_{\mathrm{A}}^{(4)}}{16 R^{4}}-\frac{9 \rho_{\mathrm{A}}^{(6)}-9 \rho_{\mathrm{B}}^{(6)}}{16 R^{6}} \\
\sigma_{\mathrm{B}}^{(0)}=\frac{9 \rho^{(0)}}{64}+\frac{63 \rho_{\mathrm{A}}^{(2)}}{64 R^{2}}+\frac{63 \rho_{\mathrm{A}}^{(4)}}{64 R^{4}}+\frac{9 \rho_{\mathrm{A}}^{(6)}-9 \rho_{\mathrm{B}}^{(6)}}{64 R^{6}} \\
\sigma_{\mathrm{A}}^{(2)}=-\frac{83 R^{2} \rho^{(0)}}{320}-\frac{21 \rho_{\mathrm{A}}^{(2)}}{20}-\frac{427 \rho_{\mathrm{A}}^{(4)}}{160 R^{2}}-\frac{62 \rho_{\mathrm{A}}^{(6)}-17 \rho_{\mathrm{B}}^{(6)}}{80 R^{4}}- \\
\frac{3 \rho_{\mathrm{A}}^{(8)}-3 \rho_{\mathrm{B}}^{(8)}}{64 R^{6}} \\
\sigma_{\mathrm{B}}^{(2)}=-\frac{97 R^{2} \rho^{(0)}}{1280}-\frac{151 \rho_{\mathrm{A}}^{(2)}}{320}-\frac{203 \rho_{\mathrm{A}}^{(4)}}{640 R^{2}}+\frac{17 \rho_{\mathrm{A}}^{(6)}+28 \rho_{\mathrm{B}}^{(6)}}{320 R^{4}}+ \\
\frac{3 \rho_{\mathrm{A}}^{(8)}-3 \rho_{\mathrm{B}}^{(8)}}{256 R^{6}}(\mathrm{~A} .11)
\end{gathered}
$$

$\mathbf{A B}_{2}$ (point group $=C_{2 v}$ )

$$
\begin{gathered}
\sigma_{\mathrm{A}}^{(0)}=\frac{\left(2 R_{\mathrm{AB}}{ }^{2}-R_{\mathrm{BB}}{ }^{2}\right) \rho^{(0)}-2 \rho_{\mathrm{A}}^{(2)}+2 \rho_{\mathrm{B}}^{(2)}}{4 R_{\mathrm{AB}}^{2}-R_{\mathrm{BB}}^{2}} \\
\sigma_{\mathrm{B}}^{(0)}=\frac{R_{\mathrm{AB}}{ }^{2} \rho^{(0)}+\rho_{\mathrm{A}}^{(2)}-\rho_{\mathrm{B}}^{(2)}}{4 R_{\mathrm{AB}}^{2}-R_{\mathrm{BB}}^{2}}
\end{gathered}
$$

$\sigma_{\mathrm{A}}^{(2)}=$

$$
\left[\left(3 R_{\mathrm{BB}}{ }^{4}-32 R_{\mathrm{AB}}{ }^{4}\right) \sigma_{\mathrm{A}}^{(0)}+\left(6 R_{\mathrm{AB}}{ }^{4}-3 R_{\mathrm{BB}}{ }^{4}\right) \rho^{(0)}+\right.
$$$$
\left.20 R_{\mathrm{AB}}{ }^{2} \rho_{\mathrm{B}}^{(2)}-10 R_{\mathrm{BB}}{ }^{2} \rho_{\mathrm{A}}^{(2)}-6 \rho_{\mathrm{A}}^{(4)}+6 \rho_{\mathrm{B}}^{(4)}\right] / 10\left(4 R_{\mathrm{AB}}{ }^{2}-R_{\mathrm{BB}}{ }^{2}\right)
$$$$
\sigma_{\mathrm{B}}^{(2)}=
$$

$$
\frac{\left(3 R_{\mathrm{BB}}{ }^{4}-32 R_{\mathrm{AB}}{ }^{4}\right) \sigma_{\mathrm{B}}^{(0)}+3 R_{\mathrm{AB}}{ }^{4} \rho^{(0)}+10 R_{\mathrm{AB}}{ }^{2} \rho_{\mathrm{A}}^{(2)}+3 \rho_{\mathrm{A}}^{(4)}-3 \rho_{\mathrm{B}}^{(4)}}{10\left(4 R_{\mathrm{AB}}{ }^{2}-R_{\mathrm{BB}}{ }^{2}\right)}
$$

$\mathbf{A B}_{3}$ (point group $=C_{3 v}$ )

$$
\begin{gathered}
\sigma_{\mathrm{A}}^{(0)}=\frac{\left(3 R_{\mathrm{AB}}{ }^{2}-2 R_{\mathrm{BB}}{ }^{2}\right) \rho^{(0)}-3 \rho_{\mathrm{A}}^{(2)}+3 \rho_{\mathrm{B}}^{(2)}}{6 R_{\mathrm{AB}}{ }^{2}-2 R_{\mathrm{BB}}{ }^{2}} \\
\sigma_{\mathrm{B}}^{(0)}=\frac{R_{\mathrm{AB}}{ }^{2} \rho^{(0)}+\rho_{\mathrm{A}}^{(2)}-\rho_{\mathrm{B}}^{(2)}}{6 R_{\mathrm{AB}}{ }^{2}-2 R_{\mathrm{BB}}{ }^{2}} \\
\sigma_{\mathrm{A}}^{(2)}= \\
{\left[\left(6 R_{\mathrm{BB}}{ }^{4}-48 R_{\mathrm{AB}}{ }^{4}\right) \sigma_{\mathrm{A}}^{(0)}+\left(9 R_{\mathrm{AB}}{ }^{4}-6 R_{\mathrm{BB}}{ }^{4}\right) \rho^{(0)}+\right.} \\
\left.30 R_{\mathrm{AB}}{ }^{2} \rho_{\mathrm{B}}^{(2)}-20 R_{\mathrm{BB}}{ }^{2} \rho_{\mathrm{A}}^{(2)}-9 \rho_{\mathrm{A}}^{(4)}+9 \rho_{\mathrm{B}}^{(4)}\right] / \\
\left.\sigma_{\mathrm{B}}^{(2)}={ }^{2}-2 R_{\mathrm{BB}}{ }^{2}\right) \\
\frac{\left(6 R_{\mathrm{BB}}{ }^{4}-48 R_{\mathrm{AB}}{ }^{4}\right) \sigma_{\mathrm{B}}^{(0)}+3 R_{\mathrm{AB}}{ }^{4} \rho^{(0)}+10 R_{\mathrm{AB}}{ }^{2} \rho_{\mathrm{A}}^{(2)}+3 \rho_{\mathrm{A}}^{(4)}-3 \rho_{\mathrm{B}}^{(4)}}{10\left(6 R_{\mathrm{AB}}{ }^{2}-2 R_{\mathrm{BB}}{ }^{2}\right)}
\end{gathered}
$$

\section{References and Notes}

(1) For a cogent alternative point of view, see ref 15.

(2) Parr, R. G.; Yang, W. Density-functional theory of atoms and molecules; Clarendon: Oxford, U.K., 1989.

(3) Mulliken, R. S. J. Chem. Phys. 1935, 3, 573.

(4) Lowdin, P.-O. J. Chem. Phys. 1950, 18, 365.

(5) Stewart, R. F.; Davidson, E. R.; Simpson, W. J. Chem. Phys. 1965, 42,3175 .

(6) Rys, J.; King, H. F.; Coppens, P. Chem. Phys. Lett. 1976, 41, 617.

(7) Stewart, R. F. Isr. J. Chem. 1977, 16, 124.

(8) Yanez, M.; Stewart, R. F.; Pople, J. A. Acta Crystallogr. A 1978, 34,641

(9) Stone, A. Chem. Phys. Lett. 1981, 83, 233.

(10) Bader, R. F. W.; Nguyen-Dang, T. T. Adv. Quantum Chem. 1981, 14,63 .

(11) Biegler-Koenig, F. W.; Bader, R. F. W.; Tang, T. H. J. Comput. Chem. 1982, 3, 317.

(12) Sokalski, W.; Poirier, R. Chem. Phys. Lett. 1983, 98, 86.

(13) Hall, G. G.; Smith, C. M. Int. J. Quantum Chem. 1984, $25,881$.

(14) Smith, C. M.; Hall, G. G. Theor. Chim. Acta 1986, 69, 63.

(15) Bader, R. F. W. Atoms in Molecules A Quantum Theory; Clarendon: Oxford, U.K., 1990

(16) Cioslowski, J.; Hay, P. J.; Ritchie, J. P. J. Phys. Chem. 1990, 94, 148.

(17) Ferenczy, G. J. Comput. Chem. 1991, 12, 913.

(18) March, N. H. Electron Density Theory of Atoms and Molecules; Academic: London, 1992.

(19) Reed, J. L. J. Chem. Educ. 1992, 69, 785.

(20) Bayly, C. I.; Cieplak, P.; Cornell, W. D.; Kollman, P. A. J. Phys. Chem. 1993, 97, 10269

(21) Bader, R. F. W. Int. J. Quantum Chem. 1994, 49, 299.

(22) Ángyán, J. G.; Chipot, C. Int. J. Quantum Chem. 1994, 52, 17.

(23) Stewart, R. F. Chem. Phys. Lett., submitted for publication.

(24) Grant, J. A.; Pickup, B. T. J. Phys. Chem. 1995, 99, 3503.

(25) Johnson, B. G.; Gill, P. M. W.; Head-Gordon, M.; White, C. A.; Maurice, D. R.; Adamson, R. D.; Adams, T. R.; Oumi, M. Q-CHEM; Q-Chem Inc.: Pittsburgh, 1996.

(26) Gill, P. M. W.; Johnson, B. G.; Pople, J. A.; Taylor, S. W. J. Chem. Phys. 1992, 96, 7178.

(27) Wang, W.-P.; Parr, R. G. Phys. Rev. A 1977, 16, 891.

(28) Slater, J. C. Phys. Rev. 1930, 36, 57.

(29) Pauling, L. The Nature of the Chemical Bond; Cornell University Press: Ithaca, NY, 1960.

(30) Wang, S. C. Phys. Rev. 1928, 31, 579.

(31) Atkins, P. W. Physical Chemistry; Oxford University Press: Oxford, U.K., 1994.

JP961524H 